\title{
Verschiedene Faserarten gleichzeitig und in Mengen schneiden
}

Das Unternehmen Schmidt \& Heinzmann hat ein neuartiges flexibles Hochleistungsfaserschneidsystem namens Fidocut (Fibre Dosing and Cutting System) entwickelt. Mit dem System sei es möglich, verschiedene Faserarten gleichzeitig und in industriellen Mengen zu schneiden. Darüber hinaus bestehe die Möglichkeit, Faserlängen von 0,1 bis 200 mm ohne Umrüstaufwand während des Schneidprozesses flexibel zu verändern. Das zu schneidende Faserspektrum umfasse Faserarten von Aramid, PE, PA, Stahl, Glas, Carbon bis Basalt oder Naturfasern, berichten die Maschinenbauer.

Das neue Faserschneidsystem wurde speziell zur Herstellung von Faserverbundwerkstoffen entwickelt. Die Schneidtechnik beruht auf dem Funktionsprinzip einer Schere, erläutern die Anlagenbauer. „Endlosfaserstränge werden mittels einer Vorschubeinheit abgezogen und über ein patentiertes Luftfördersystem, das die Fasern zwischen Vorschubein- heit und Schneide unter Spannung hält, dem Faserschneidesystem zugeführt", heißt es. Das Schneidsystem bestehe aus einem rotierenden Messer und drei feststehenden Gegenklingen. Die Fasern treten vor den Schneiden an der Gegenklinge durch ein Fadenauslassmodul aus, bevor sie durch einen ziehenden Schnitt des an der hochpräzisen Schneidspindel befestigten rotierenden Messers geschnitten werden. Damit werde ein kontinuierliches Schneiden ermöglicht.

Die Spindel wird mit einem Regelantrieb mit bis zu 18.000/min angetrieben. Faservorschübe von bis zu $300 \mathrm{~m} / \mathrm{min}$ sind laut Schmidt \& Heinzmann möglich. Zur Sicherstellung eines konstanten Schneidspalts zwischen der rotierenden Messerklinge und den drei fixen Gegenklingen werde der gesamte Schneidraum mittels eines Wasser-/Luftkreislaufs temperiert. Durch das Prinzip des Scherenschnitts könnten auch sehr zähe oder weiche Fasern geschnitten werden.
Die Firma wurde 1949 gegründet und produziert Maschinen und Anlagen zur Herstellung faserverstärkter Kunststoffe. Für ihr Hochleistungsfaserschneidsystem ist das Unternehmen aus Bruchsal als einer von fünf Preisträgern mit dem Innovationspreis des Landes Baden-Württemberg 2012, dem Dr.-Rudolf-Eberle-Preis, ausgezeichnet worden.

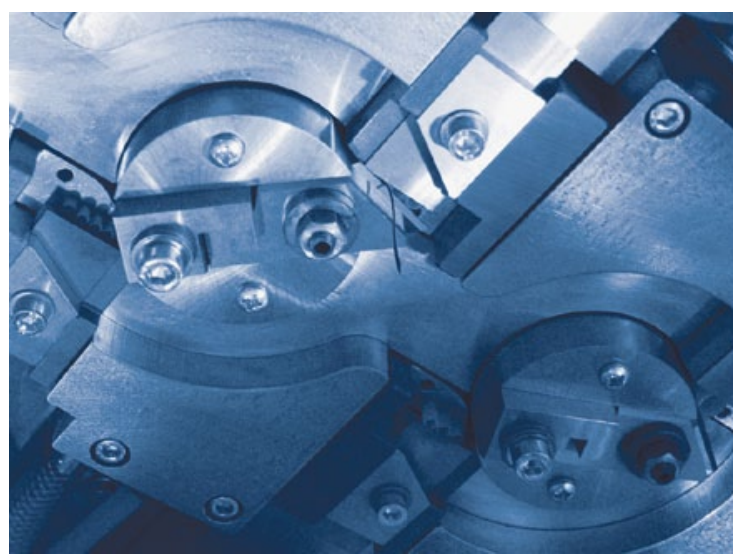

FÜR DIE LUFTFAHRT

IPF DRESDEN UND EAST-4D WOLLEN TAILORED FIBER PLACEMENT WEITERENTWICKELN

Das Leibniz-Institut für Polymerforschung Dresden (IPF) und das Dresdner Unternehmen der East-4D Carbon Technology haben eine Kooperationsvereinbarung zur Weiterentwicklung der Tailored -Fiber-PlacementTechnik (TFP) und deren Nutzung zur Entwicklung von extrem belastbaren Hochleistungsbauteilen für den Luftfahrtsektor unterzeichnet.

Im Rahmen der Vereinbarung sollen erstmalig die exakten Faserpositionen mittels TFPTechnik gefertigter Bauteile komplett in die Berechnung einfließen. Die virtuelle Gestaltung und Berechnung dieser zum Teil hochgradig komplexen Faser-Kunststoff-Verbundbauteile erfolgt in einem neuartigen Ansatz abseits existierender Berechnungsnormen und Standards. Dieses Vorgehen soll neue Anwendungsperspektiven eröffnen. Gemeinsam erwarten die Kooperationspartner, dass sich die Eigenschaftspotenziale der Kohlenstofffasern deutlich besser ausschöpfen lassen. Gerade für extreme Anwendungen - etwa für die nächste Generation sehr schnell drehender Bauteile von umweltschonenden Flugzeugtriebwerken - sei die Beherrschung der exakten Faserpositionen ausschlaggebend im Design- und Herstellungsprozess. Die Dresdner Experten aus Forschung und Industrie wollen gemeinsam die Berechenbarkeit dieser in TFP-Technik gefertigten Luftfahrtstrukturen ermöglichen. Dadurch sollen dann auch ext- rem sicherheitskritische Bauteile bis zu ihrem Einsatzlimit belastet werden können.

Die TPF-Technik wurde bereits in den 1990er Jahren am IPF entwickelt. Sie erlaubt über die sticktechnische Ablage von Verstärkungsfasern die Herstellung von Bauteilen, in denen durch eine konsequent beanspruchungsgerechte Anordnung der Fasern maximale mechanische Eigenschaften bei geringer Masse erreicht werden können. Parallel zu diesen Aktivitäten wurden in dem Dresdner Unternehmen East-4D Carbon Technology Bauteile aus Kohlenstofffasern berechnet und etwa mit der Fertigungstechnik des Wickelns von Kohlenstofffasern in den Markt eingeführt. 